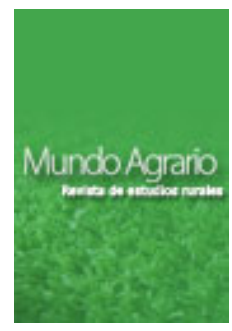

Mundo Agrario vol. 18, nº 38, e054, agosto 2017. ISSN 1515-5994

Universidad Nacional de La Plata.

Facultad de Humanidades y Ciencias de la Educación.

Centro de Historia Argentina y Americana

\title{
Transformaciones territoriales en ámbitos rurales del Noroeste Argentino y la Región Pampeana hacia finales del siglo XX e inicios del XXI
}

\author{
Territorial transformations in rural areas of the Argentine Northwest \\ and The Pampean Region towards the end of the XX century and \\ beginnings of the XXI
}

\author{
Ana Garay *, Julieta Krapovickas *, Claudia Mikkelsen ** \\ * Instituto Superior de Estudios Sociales, CONICET, Universidad Nacional de Tucumán, Argentina, ** \\ CONICET - Universidad Nacional de Mar del Plata - Universidad Nacional del Centro de la Provincia de \\ Buenos Aires, Argentina | la garay@hotmail.com, krapovickasjulieta@gmail.com, \\ claudiamikkelsen@gmail.com
}

\section{PALABRAS CLAVE}

Transformaciones territoriales

Ámbitos rurales

Noroeste Argentino

Región Pampeana

\section{RESUMEN}

Desde las últimas décadas del siglo XX, los sectores rurales argentinos son escenario de profundas transformaciones socioproductivas ligadas a la expansión de las actividades económicas intensivas en el uso de capital. Entre ellas podemos mencionar la agricultura para exportación, la vitivinicultura y la minería. Tanto en el Noroeste Argentino (NOA) como en la Región Pampeana, estas actividades productivas, así como los procesos de acaparamiento, privatización y concentración de la tierra son particularmente visibles. El objetivo del artículo es analizar las transformaciones territoriales del campo argentino comparando los procesos en el NOA y en la Región Pampeana entre la última década del siglo XX y la primera del siglo XXI. El estudio involucra a diez provincias, que comprenden el $35 \%$ de la superficie del país, cuentan con 170.000 explotaciones agropecuarias e incluyen al $60 \%$ de la población rural del país.

\section{KEYWORDS ABSTRACT}

Territorial transformations

Rural areas

Northwest Argentina

Pampas Region
Since the last decades of the twentieth century, Argentine rural areas are the scene of deep socio-productive transformations linked to the expansion of intensive economic activities in the use of capital. Among them, we can mention agriculture for export, viticulture, and mining. In both cases, the Argentine Northwest (NOA) and the Pampean Region, these productive activities, as well as land grabbing processes and the privatization and concentration of land, are particularly visible. The aim of the paper is to analyze the territorial transformations of the Argentinian's rural areas, comparing the processes in the NOA and in the Pampean Region between the last decade of the $20^{\text {th }}$ century and the first of the 21 st century. The study involves ten provinces, which comprise $35 \%$ of the land area, have 170,000 farms, and include $60 \%$ of the rural population. 


\section{Introducción}

A pesar de los avances ocurridos en los últimos 15 años, actualmente América Latina continúa siendo una de las regiones más desiguales del planeta (sólo superada por el África Subsahariana) (PNUD, 2010), ya que las fuentes de riqueza en las diferentes naciones no se han distribuido equitativamente, ni mucho menos popularizado (Kay, 2007; Amarante y Jiménez, 2015). En todo caso, lo que sí ha mejorado es la distribución del ingreso, pero no de la riqueza, cuya base estructural se mantiene intacta (Bárcena y Prado, 2016; CEPAL, 2016).

La exportación de recursos naturales sigue siendo una de las actividades fundamentales para el crecimiento económico y el aumento de los ingresos de los estados latinoamericanos, ya que fortalece el modelo económico imperante en las décadas de mayor auge de neoliberalismo económico y nos enfrenta, a lo largo de las últimas décadas, a un nuevo boom extractivista en la región (Bebbington y Humphreys-Bebbington, 2011).

Dicho modelo, además de generar importantes costos y riesgos ambientales (para las poblaciones rurales fundamentalmente), profundiza las desigualdades y asimetrías sociales en lugar de reducirlas (Reboratti, 2007; Göbel et al, 2014). Con este sistema la tierra se privatiza y extranjeriza a ritmos vertiginosos (Borras Jr. et al, 2012), lo cual concentra aún más la riqueza y excluye al grueso de la población de los beneficios del uso de los bienes naturales comunes (Krapovickas et al, 2016).

En sintonía con el contexto latinoamericano, desde las últimas décadas del siglo XX, los espacios rurales argentinos vienen siendo escenario de profundas transformaciones socioproductivas ligadas a la expansión de las actividades económicas intensivas en el uso de capital, que pueden caracterizarse como extractivas. Entre ellas podemos mencionar la agricultura para exportación (por ejemplo, de soja, citrus, olivos), la vitivinicultura y la minería (Svampa y Antonelli, 2009; Massuh, 2012; Giarracca y Teubal, 2013; Krapovickas, 2016).

Los gobiernos insisten en las ventajas del modelo que, con base en la renta extractivista, permite la expansión de derechos y la reducción de los niveles de pobreza mediante la aplicación de una batería de políticas sociales (Svampa, 2011). Sin embargo, tanto en el Noroeste Argentino (NOA) como en la Región Pampeana son particularmente visibles las consecuencias de estas actividades productivas capital-intensivas en la calidad de vida de la población (Velázquez, 2016), a la cual se excluye mediante procesos de acaparamiento, privatización y concentración de la tierra (Rivas y Natera-Rivas, 2009; Gras 2012; Morina y Cacace, 2010; Reboratti, 2006), con su correlato de despojo y pérdida de medios de vida para las poblaciones campesinas e indígenas (Bolsi y Paolasso, 2009) así como para pequeños productores chacareros (Balsa, 2006; Gras, 2006; Domínguez y Sabatino, 2006; Shmite, 2008).

Considerando esto, nos preguntamos cuál es la dimensión de este fenómeno en ambas regiones y cuáles son las similitudes y diferencias. Por tanto, el artículo tiene como objetivo analizar las transformaciones territoriales del agro argentino actual comparando los procesos en el Noroeste Argentino y la Región Pampeana hacia finales del siglo XX e inicios del siglo XXI. Se investigan algunas variables centrales de la estructura agraria en ambas regiones, en pos de visibilizar la persistencia de las desigualdades en el interior y entre los territorios estudiados. Consideramos que es importante esta comparación ya que se trata de las dos regiones más contrapuestas del país, pese a lo cual se pueden observar procesos y tendencias similares.

\section{Globalización y desarrollo geográfico desigual}

Los desarrollos teóricos y las conceptualizaciones sobre las consecuencias sociales y territoriales de la globalización son aportes importantes para nuestro trabajo, particularmente el enfoque del desarrollo geográfico desigual, caracterizado por Harvey (2003). Para este autor, la globalización es un proceso de desarrollo temporal y geográfico desigual (2003), en el que las diferencias geográficas son resultantes de legados históricos y geográficos, y se sostienen por los fenómenos político-económicos y socioecológicos que tienen lugar en el presente. El proceso “concentra la riqueza y el poder y más oportunidades políticas y económicas en unas cuantas localizaciones selectivas y dentro de unos cuantos estratos restringidos de población” (Harvey, 2003, p. 102). Se 
pone así de manifiesto la "vulnerabilidad selectiva" que expone de manera desigual a las poblaciones a efectos tales como el desempleo, la degradación de los niveles de vida y la pérdida de recursos y de calidades ambientales.

Asimismo, la lógica de la movilidad del capital descripta por Smith (2006) se asocia perfectamente con la teoría del desarrollo geográfico desigual. Para Smith el capital se mueve a lugares específicos donde puede extraer ventajas económicas y lograr tasas de ganancia más elevadas. En búsqueda de ganancias y obligado a competir, el capital se concentra, no sólo en los bolsillos de algunos por encima de los de otros, sino también en ciertos lugares a expensas de otros. En el mismo sentido, Harvey (2004) plantea la teoría de la "solución espacial” (spatial fix) a la crisis. Frente a la tendencia a la crisis que el capitalismo genera, el capital desarrolla estrategias espaciales y temporales precisas que logran prorrogarla. Las soluciones o ajustes espaciales serían en principio dos: la reorganización espacial de un territorio o región económica y la exportación del capital y el trabajo sobrantes más allá de la región en la que se han generado. Harvey refiere a una solución temporal, que implicaría un desplazamiento hacia el futuro de la crisis mediante el crédito y las finanzas. En consecuencia, la diferenciación de lugares, se explica cada vez menos por una cuestión de locación y dotación natural y en su lugar lo que impera es la lógica espacial inherente al capital.

\section{Metodología}

El estudio se realizó sobre la población rural (agrupada y dispersa) de las diez provincias que integran las regiones $\underline{1}$ Pampeana (Buenos Aires, Córdoba, Entre Ríos, La Pampa, Santa Fe) y NOA (Jujuy, Salta, Tucumán, Santiago del Estero y Catamarca), las cuales representan al 34,7 \% de la superficie del país (1.298.304 km²) y tenían en 2008, 168.593 explotaciones agropecuarias (EAPs) según el Censo Nacional Agropecuario (CNA) 2008 -aproximadamente el 60 \% del total de EAPs del país-. La población rural involucrada es de 2.175.911 personas, que representan el 60,4 \% de la población rural del país, y el 5,4 \% del total de la población argentina en 2010 (INDEC).

Para analizar la estructura agraria y productiva se indagaron cuatro variables:

1) Cantidad de explotaciones agropecuarias, 2) EAPs con y sin límites definidos, 3) tamaño medio de las EAPs, y 4) composición de las exportaciones provinciales. Se utilizaron los tres últimos censos nacionales agropecuarios 1988, 2002 y 2008, como así también datos del Sistema Integrado de Información Agropecuaria (SIIA). Si bien inicialmente se consideró no utilizar los datos del último censo nacional agropecuario (2008) debido a la mala calidad -ya que el relevamiento no fue completado en algunas provincias-, finalmente se decidió explorar y analizar esta fuente evaluando críticamente el comportamiento de los datos y considerando su evolución histórica ${ }^{2}$. La variable composición de las exportaciones provinciales nos permitió analizar los procesos de avance del extractivismo y la consecuente reprimarización de las economías regionales $\underline{3}$.

\section{La argentina rural, un zoom en el Noroeste Argentino y la Región Pampeana}

En Argentina, y gran parte de Latinoamérica, durante la década de los noventa se instauró un modelo económico caracterizado por la apertura económica, el cual generó hondas transformaciones. Sin duda, el sector agropecuario argentino no resultó ajeno al conjunto de cambios estructurales que se produjeron y en este proceso se desmontaron "la mayoría de los organismos y normas que permitieron llevar adelante una política sectorial por más de medio siglo” (Lattuada y Neiman, 2005, p. 9). El resultado de la aplicación de tales políticas implicó, por un lado, la instauración definitiva de la revolución verde en Argentina, con incorporación de tecnologías de procesos, es decir, innovaciones intangibles inmateriales comprendidas básicamente por la información y el conocimiento (Blanco 2005), con la consiguiente modernización tecnológica, duplicación de la producción y aumentos en el volumen de las exportaciones agrícolas. Por otro lado, sin embargo, aumentó la concentración de la tierra, se redujo el empleo de mano de obra asalariada (Blanco, 2005; Neiman 2010) y se transformó la 
estructura social agraria excluyendo a los pequeños y medianos productores (Lattuada y Neiman, 2005; Blanco, 2005).

De esta manera, los cambios en la política económica argentina permitieron (o más bien estimularon) el avance del agronegocio, con su correlato de agriculturización (Barsky y Gelman, 2005) y sojización. En efecto, si tomamos por caso el cultivo de soja que actualmente se expande en gran parte del territorio nacional (especialmente en la Región Pampeana pero también en el NOA) se observa que tal avance tiene dos consecuencias importantes para el perfil del agro argentino: en primer lugar, contribuye significativamente a la desaparición de una serie de explotaciones agropecuarias y, en segundo lugar, se trata de una producción orientada casi exclusivamente hacia la exportación, por lo cual afecta la producción de alimentos básicos orientados hacia la demanda del sector interno (Giarracca y Teubal, 2006). Tal como expresa Giberti:

La Argentina, caracterizada por una clase media agraria casi inexistente en el resto de América Latina, ha sufrido una acentuada merma de explotaciones medianas y pequeñas. Entre 1988 y 2002 desaparecieron en promedio unas 20 explotaciones por día, casi todas de tales estratos. Tan abultada cifra no obedece al esperable aumento de tamaño que la tecnificación exige a las explotaciones medianas, sino a que la gran empresa avanzó insanamente sobre las demás. (2003, p. 106)

La observación de los datos provenientes de los CNA permite confirmar esta tendencia a nivel nacional donde los veinte años que transcurrieron entre 1988 y 2008 (fecha del último censo nacional agropecuario) desaparecieron casi 145 mil EAPs, a razón de 7200 por año (Tabla 1). Si bien (lógicamente por la cantidad de años analizados en cada período) en los primeros 14 años es cuando se concentra la mayor pérdida absoluta de EAPs, es entre 2002 y 2008 (los últimos 6 años analizados) cuando se acelera la velocidad de la reducción, y desaparecen unas 9500 unidades productivas por año. Los procesos de intensificación productiva, la expansión urbana y las propias características del paquete tecnológico explican la vulnerabilidad selectiva en la que ingresan los pequeños productores y los campesinos, lo que se visibiliza en la reducción de la superficie total de las EAPs, aunque no en los niveles y ritmos que se observan en valores absolutos.

Tabla 1

\section{Cantidad y superficie de las EAPs, valores absolutos y variaciones relativas, en porcentajes (1988,} 2002, 2008). Argentina

\begin{tabular}{|c|c|c|c|c|}
\hline & Cantidad & SuperficieHas & $\begin{array}{c}\text { variación } \\
\text { cantidad(1988 =100) }\end{array}$ & $\begin{array}{c}\text { variación superficie(1988 } \\
\mathbf{1 0 0})\end{array}$ \\
\hline 1988 & 421.221 & 177.437 .398 & 100,0 & 100,0 \\
\hline 2002 & 333.533 & 174.808 .564 & 79,2 & 98,5 \\
\hline 2008 & 276.581 & 155.424 .819 & 65,7 & 87,6 \\
\hline
\end{tabular}

Fuente: Censos Nacionales Agropecuarios (CNA) 1988, 2002 y 2008.

Los cambios aplicados en la etapa de apertura económica han tenido un fuerte impacto en la argentina rural, con efectos no sólo en la producción agrícola sino también en la estructura agraria, en la tenencia de la tierra, en las formas de habitar los espacios rurales, en la movilidad espacial y en el trabajo agrario, lo cual tiene especial relación con la teoría de la solución espacial de Harvey (Harvey, 2004). En paralelo con el avance de la revolución verde, se observa una situación de ajuste espacial, con exportación del trabajo sobrante; una solución temporal basada en una estrategia espacial de movilidad de los sujetos desde las áreas rurales hacia las áreas urbanas, en donde es posible que se empleen como prestadores de servicios al propio sector agropecuario (tercerización). Resultado de estos procesos, en la actualidad se observa que lo rural excede lo agropecuario debido a que cada vez más las poblaciones rurales se vuelcan al sector de servicios, de modo que asistimos a una progresiva “desagrarización” de las ocupaciones de las poblaciones que habitan los espacios rurales. Por otro lado, a pesar de 
la relativa homogeneidad del proceso comentado en todo el país, las transformaciones en el sector agrario y rural fueron sensiblemente diferentes entre las regiones argentinas. Así, por ejemplo -y retomando el caso de la soja-, la aplicación de un mismo paquete tecnológico (centrado en el uso de biotecnología, semillas transgénicas, siembra directa y gran cantidad de agroquímicos) en territorios tan diversos como la Región Pampeana y el NOA generó efectos territoriales y demográficos disímiles. De este modo, mientras que en gran parte de la Región Pampeana el cambio en el paradigma productivo significó el reemplazo de cultivos tradicionales como el trigo y el girasol y la virtual desaparición de la rotación de agricultura y ganadería, en amplios sectores del NOA (antes considerados marginales para el desarrollo de la agricultura), la expansión agrícola y la ganadería capitalizada significó la deforestación de enormes superficies de bosques nativos, especialmente en las regiones del parque chaqueño ${ }^{4}$ y sobre el pedemonte de la selva tucumano-oranense (García et al, 2016), así como el desplazamiento de las poblaciones que las habitaban (Reboratti et al 1996; Krapovickas 2009; Román et al, 2016). Consideramos esto último de suma importancia ya que la frontera no avanzó sobre territorios vacíos, sino poblados por familias con una racionalidad no ligada con esta expansión (De Dios, 2006; citado por Román et al, 2016), las cuales históricamente se han enfrentado a los procesos de desalojo y despojo de la tierra $\underline{5}$.

En este contexto, la diferencia fundamental entre las regiones analizadas es lo que se podría llamar su tradición capitalista (Bolsi, 1997). Así pues, la Región Pampeana, a diferencia del NOA, se caracteriza por su antigua inserción capitalista, la cual se filtró en sus principales procesos productivos, lo que resultó en un desarrollo social y económico que en mucho se asemeja al típico de zonas similares de las economías centrales desarrolladas (Manzanal, 1995; Barsky y Gelman, 2005). Ante esto, podemos decir que la Pampa argentina se ha transformado en una copia tardía de la pradera estadounidense dado que, tanto aquí como allí, la mecanización y la motorización insertas en una economía agrícola puramente especulativa han dado lugar a la creación de centros de servicio, que ya no son pueblos sino pequeñas ciudades activas (Gaignard, 1989, p. 46). Por su parte, en la región del NOA, si bien estos procesos también tuvieron lugar, las lógicas fueron diferentes y la inserción se concretó a la cola de la producción pampeana de un modo tardío y marginal. Recién será a partir de la década de 1990, cuando - a partir del incremento de los precios internacionales de algunos productos alimenticios (cereales y oleaginosas especialmente) y del petróleo- se hizo rentable la producción en áreas que otrora se consideraban desfavorables por las características de sus suelos y clima; por ello, desde las últimas décadas del siglo XX, en el sector rural del NOA, se expandieron las actividades productivas primarias orientadas al mercado externo (Osatinsky, 2009; Rivas y Natera Rivas, 2009; Rivas, 2013). En este sentido, también se ha hablado de un proceso de "pampeanización” productiva de ciertas regiones del NOA, particularmente aquellas en las que se constató un cambio en la utilización de los factores de producción: mayor inversión de capital, utilización de mano de obra más especializada y por período corto, movilidad de los medios de producción y por lo tanto sin necesidad de grandes infraestructuras rurales, mayor hincapié en la mano de obra asalariada y sin relaciones de patronazgo. Todo esto ha llevado a que la nueva estructura agraria se asemeje a las típicas de la región pampeana. De esta forma, la frontera agraria es una frontera vacía de hombres en el campo y llena de maquinaria y tecnología, que depende de pequeñas ciudades que le sirven de centro de servicios (Reboratti, 1996, p. 165)

\section{Análisis comparativo de la estructura agraria del Noroeste Argentino y la Región Pampeana}

\subsection{Evolución de las explotaciones agropecuarias}

En las regiones analizadas se puede verificar la evolución a la baja en la cantidad de EAPs que constatamos en la sección anterior a nivel nacional.

En la Región Pampeana, durante el primer intercenso (1988 - 2002), la disminución fue de aproximadamente 55 mil EAPs, y entre 2002 y 2008 fue de 20 mil EAPs. Las provincias más afectadas fueron Córdoba y Buenos Aires entre 1988 y 2002, y nuevamente Buenos Aires y Entre Ríos entre 2002 y 2008 (tabla 2). En el último intercenso (2002 - 2008) debe destacarse que las provincias de Córdoba y La Pampa presentan un incremento, si bien modesto, en el número total de EAPs, que rompe la tendencia del primer intercenso en el que todas las provincias 
mostraron marcadas reducciones.

\section{Tabla 2}

\begin{tabular}{|c|c|c|c|c|c|c|}
\hline \multirow{2}{*}{$\begin{array}{c}\text { Evolución de la cantidad de explotaciones agropecuarias, valores absolutos y variaciones relativas, en } \\
\text { porcentaje (1988, 2002, 2008). Región Pampeana y NOA }\end{array}$} \\
\hline \multirow{2}{*}{ Provincias } & \multicolumn{3}{|c|}{ Datos absolutos } & \multicolumn{3}{c|}{ Variaciones relativas (\%) } \\
\cline { 2 - 7 } & $\mathbf{1 9 8 8}$ & $\mathbf{2 0 0 2}$ & $\mathbf{2 0 0 8}$ & $\mathbf{1 9 8 8 - 2 0 0 2}$ & $\mathbf{2 0 0 2 - 2 0 0 8}$ & $\mathbf{1 9 8 8 - 2 0 0 2}$ \\
\hline Región Pampeana & 191.280 & 136.799 & 116.963 & $-28,48$ & $-14,5$ & $-38,9$ \\
\hline Buenos Aires** & 75.531 & 51.116 & 34.675 & $-32,32$ & $-32,16$ & $-54,1$ \\
\hline Córdoba** & 40.817 & 26.226 & 27.084 & $-35,75$ & 3,27 & $-33,7$ \\
\hline Entre Ríos** & 27.197 & 21.577 & 18.282 & $-20,66$ & $-15,27$ & $-32,8$ \\
\hline La Pampa** & 8.718 & 7.775 & 7.940 & $-10,82$ & 2,12 & $-8,9$ \\
\hline Santa Fe* & 37.029 & 28.103 & 26.974 & $-24,11$ & $-4,02$ & $-27,2$ \\
\hline NOA & 64.986 & 59.257 & 51.630 & $-8,8$ & $-12,9$ & $-20,6$ \\
\hline Catamarca* & 9.538 & 9.138 & 9.012 & $-4,2$ & $-1,4$ & $-5,5$ \\
\hline Jujuy* & 8.526 & 8.983 & 8.733 & 5,4 & $-2,8$ & 2,4 \\
\hline Salta* & 9.229 & 10.297 & 10.317 & 11,6 & 0,2 & 11,8 \\
\hline Santiago del Estero* & 21.122 & 20.949 & 15.899 & $-0,8$ & $-24,1$ & $-24,7$ \\
\hline Tucumán** & 16.571 & 9.890 & 7.669 & $-40,3$ & $-22,5$ & $-53,7$ \\
\hline * Provincia con superficie censada en 2008 superior al 95 \% de la del CNA 2002 & & \\
\hline ** Provincias con superficie censada en 2008 inferior al 95 \% de la del CNA 2002 & \\
\hline
\end{tabular}

Fuente: CNA 1988, 2002 y 2008

El caso del NOA, a diferencia de la Región Pampeana, se debe analizar a la luz de los marcados contrastes en su paisaje natural. Las áreas montañosas y los desiertos ocupan una proporción destacada de la superficie regional y los suelos aptos para la agricultura son, en términos absolutos y relativos, menores que los de la Pampa argentina. Sin embargo, esa variedad de paisajes no se refleja en una gran diversidad económico-productiva, ya que la economía regional sigue estando conducida por las producciones primarias, las cuales se asientan en una estructura agraria históricamente desequilibrada (Bolsi, 1997) que responde al tipo bimodal, con fuerte presencia campesina y un alto porcentaje de explotaciones sin límites definidos, muy similar a la estructura agraria de cualquiera de los países latinoamericanos como Bolivia, Colombia, Ecuador o Perú (Paz y Jara, 2014).

Al considerar entonces, en primer lugar, el total de EAPs en las dos décadas analizadas constatamos en la región del NOA una notable disminución en su número absoluto. Mientras en 1988 el total de EAPs llegaba casi a 65 mil unidades, en 2008 las mismas apenas superaban las 50 mil (tabla 2). Las provincias que mayor reducción mostraron en el número de EAPs son Tucumán y Santiago del Estero, en las cuales la variación relativa fue de 53.7 \% y 24.7 \% respectivamente. En esta última pasaron de 21.000 a aproximadamente 16.000 y en Tucumán el número de EAPs pasó de 16.500 a menos de la mitad (7.600). Catamarca, por su parte, mostró una reducción leve en la cantidad de EAPs. En contraposición, en Jujuy y Salta la cantidad de EAPs se incrementó en el período considerado. En el primer caso, el incremento fue de apenas un 2,4 \%, mientras en el segundo ascendió al 11,8 \%.

Las dos regiones analizadas muestran similitudes y diferencias. En primer lugar, destacamos la cantidad de EAPs consideradas en cada caso, ya que mientras la Región Pampeana involucra al 42 \% de las EAPs argentinas hasta el 2008 (117 mil EAPs), podemos observar que la cantidad de EAPs en el NOA en el mismo año era menos de la mitad (50 mil EAPs, las cuales representan un 18 \% de las EAPs argentinas). En segundo lugar, la magnitud de los cambios ha sido diferente en cada región en términos absolutos y relativos; la disminución de las EAPs ha sido mucho más intensa en la Región Pampeana en los 20 años analizados, -38,8 \% frente a una tasa de variación de $-20 \%$ en el NOA. 
Otra diferencia sustancial se da por la homogeneidad del proceso en cada región, ya que mientras en la Región Pampeana todas las provincias tienen una evolución muy similar (con algunos contrastes hacia el último intercenso), en el NOA encontramos importantes diferencias entre las provincias.

Finalmente, se debe destacar la discrepancia en la temporalidad de los procesos operados en las dos regiones de estudio. Si bien las mismas tendencias se repiten en ambas, en la Región Pampeana observamos que, durante el primer intercenso, la reducción de las EAPs fue más intensa (se perdían casi 4000 EAPs por año entre 1988 y 2002, y 3300 entre 2002 y 2008), en cambio en el NOA se encuentra un retraso en el proceso de disminución de unidades productivas, ya que será en el segundo intercenso cuando se observen las mayores reducciones (400 EAPs menos por año entre 1988 y 2002 frente a una pérdida de 1300 EAPs por año entre 2002 y 2008); esto se explica por la ampliación de la globalización en sistemas temporales desfasados entre las regiones en estudio, hecho que reitera su temprana actuación en la Región Pampeana y su arribo más tardío al NOA.

\subsection{Evolución de las explotaciones agropecuarias con límites definidos y sin límites definidos}

En relación con la evolución de la cantidad de EAPs con límites definidos y sin límites definidos, las diferencias en las regiones analizadas se profundizan. En la tabla 3 podemos observar que, si bien en la Región Pampeana estas EAPs no representan un número tan significativo como en el NOA, es interesante señalar su reducción a lo largo del período en estudio. De este modo, Córdoba es la que muestra mayor presencia de unidades sin límites definidos, seguida de La Pampa y Santa Fe. En el caso de Córdoba, sin embargo, se encuentra una evolución hacia la reducción de este tipo de unidades productivas, mientras que las otras dos provincias señaladas presentan aumentos significativos en los 20 años analizados. No obstante, destacamos la importante diferencia entre los datos de los censos 2002 y 2008, lo que hace suponer una subestimación en el registro del 2002.

$\underline{\text { Tabla } 3}$

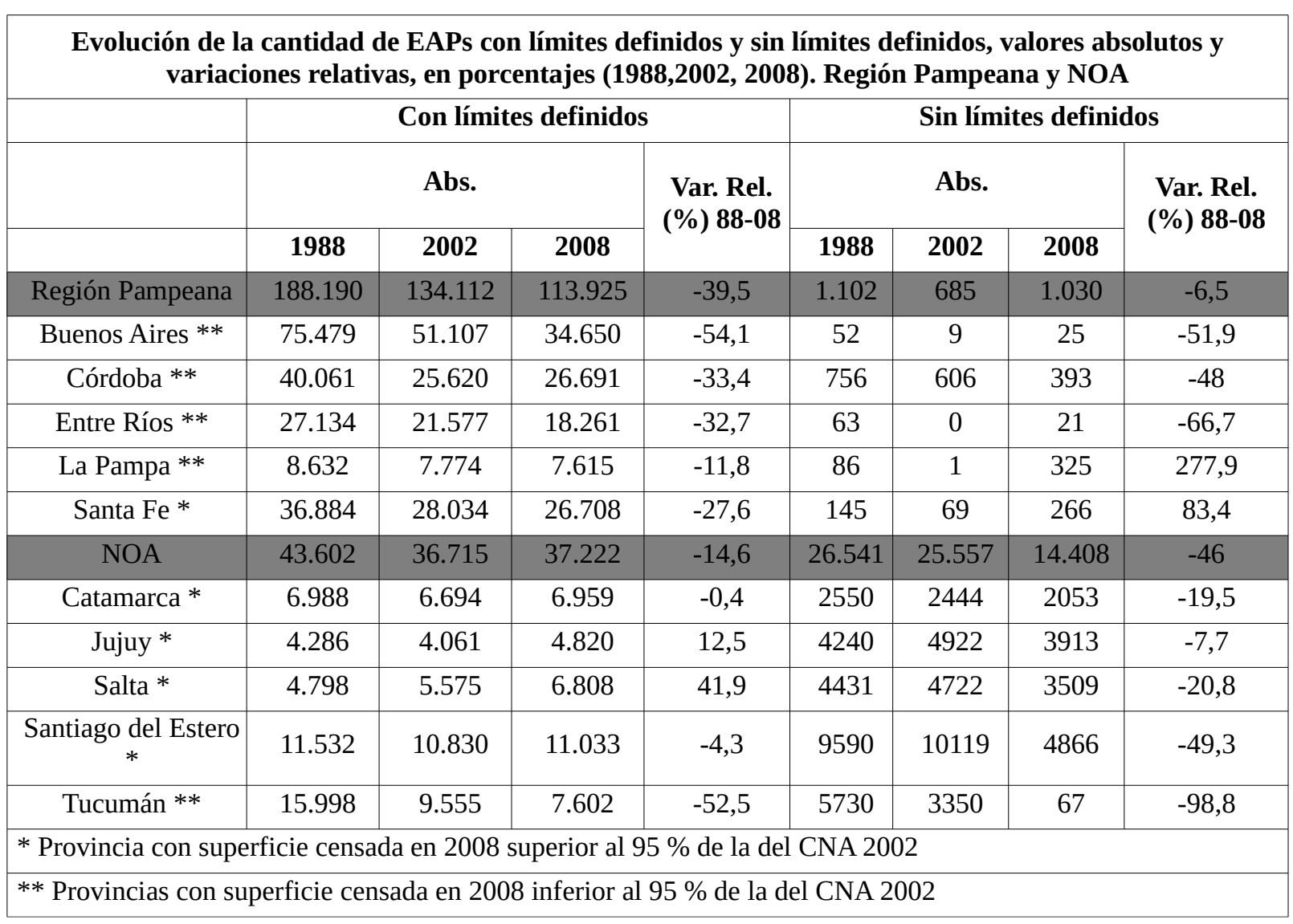

Fuente: CNA 1988, 2002 y 2008. 
Respecto a la evolución de esta variable para la región del NOA, en la misma tabla vemos que es la disminución de las EAPs sin límites definidos la tendencia más fuerte tanto a nivel regional como provincial. Entre 1988 y 2008, la región en su conjunto perdió unas 6.000 EAPs con límites definidos, pero la reducción de las EAPs sin límites definidos fue mucho más importante, ya que implicó la desaparición de unas 12.000 unidades. Otra diferencia en la evolución de las EAPs con límites definidos y sin límites definidos es que mientras en las primeras el descenso fue muy marcado sólo en la provincia de Tucumán, en el caso de las segundas, todas las provincias mostraron una tendencia a la reducción de estas unidades. En efecto, las EAPs con límites definidos aumentaron en Salta y Jujuy, y prácticamente se mantuvieron invariables en Catamarca y Santiago del Estero. En cambio, las EAPs sin límites definidos (otrora muy importantes en la región, dada la importancia de los modos campesinos de producción) se redujeron drásticamente en el período analizado en todas las provincias, a excepción de Jujuy, donde el descenso fue menos abrupto. Un aspecto característico de las explotaciones sin límites definidos es la de tener límites imprecisos o no tenerlos; los regímenes de tierra relacionados con este tipo de explotaciones son los de ocupantes de tierras privadas y fiscales, campos comuneros, aparcerías precarias y sucesiones indivisas, en las que el campesinado se constituye como el principal poblador. En este sentido, se presenta una situación de precariedad en cuanto a la falta de títulos de dominio legal de la tierra, inseguridad jurídica que agrava la situación frente al proceso de expansión de la frontera agropecuaria (Paz y Jara, 2014) y expone a las poblaciones a una situación de vulnerabilidad ante el avance del capital.

De esta manera, en la región NOA, las EAPs sin límites definidos pasaron de representar en 1988 el 33 \% del total de las unidades productivas a representar el $28 \%$ en 2008. Lo curioso es que, en 2002, si bien estas explotaciones disminuyeron, la caída de las EAPs con límites definidos fue más abrupta, por lo que la proporción de las primeras aumentó significativamente. En ese año, el 38 \% de las EAPs no tenían sus límites definidos. La reducción, entonces, de la proporción de estas EAPs sobre el total se concreta durante los últimos 6 años analizados (2002-2008), lo que nos da una idea de la magnitud, rapidez e intensidad del cambio operado en la región.

Si hacemos una síntesis de las diferencias encontradas entre las dos regiones, podemos mencionar las evidencias en torno a las magnitudes consideradas en cada caso. Así pues, en el NOA, las EAPs sin límites definidos son muy importantes aún en 2008 (en términos absolutos y relativos), con 14 mil unidades frente a las 1000 de la Región Pampeana. Otra diferencia viene dada por la heterogeneidad en las tendencias en el interior de las regiones y en la temporalidad de los procesos. De este modo, entre 1988 y 2002, las EAPs sin límites definidos aumentaron en 3 provincias del NOA y entre 2002 y 2008 la tendencia hacia una reducción sostenida de estas unidades se homogeneiza en la región con caída de los valores en todas las provincias. También las EAPs con límites definidos tienen una evolución muy dispar entre las provincias del NOA. Encontramos mayor homogeneidad en la evolución de las EAPs con límites definidos o sin límites definidos en la Región Pampeana, donde la cantidad de EAPs con límites definidos cae o se mantiene y, en el caso de las EAPs sin límites definidos, cuesta marcar una tendencia, dada la subestimación aparente de los datos en 2002.

\subsection{Tamaño medio de las explotaciones agropecuarias}

Otra de las variables analizadas en este trabajo para describir la evolución de la dimensión estructura agraria es el tamaño medio de las EAPs. Respecto a esta variable, observamos que durante los períodos intercensales analizados conjuntamente con la reducción generalizada en el número de EAPs aumenta su tamaño medio. Así pues, en la Región Pampeana, el tamaño medio que se incrementa en el primer período de análisis (1988-2002) -pasa de 370 ha a 500 ha- se mantiene para el segundo intercenso (2002-2008) en 486 ha, lo que implica una variación 1988/2008 de 31,4 \%. A nivel provincial, denota reducciones abruptas en La Pampa, leves en el caso de Córdoba, y de estabilidad para Entre Ríos, Santa Fe y Buenos Aires (gráfico 1)

Por su parte, en el NOA, la tendencia durante las dos décadas analizadas fue hacia un leve incremento de la superficie media, fundamentalmente marcado entre los dos primeros censos (1988-2002). De esta manera, el tamaño medio de las EAPs pasó de 270 hectáreas en 1988 a 326 ha en 2008, con una variación 1988/2008 de 21,3 
\%. La situación en el interior de la región, sin embargo, presenta marcados contrastes: algunas provincias como Salta y Jujuy se separan de la tendencia general mostrando una fuerte reducción en las superficies de sus EAPs y otras, como Tucumán, Catamarca y Santiago del Estero, presentan los incrementos más notables en los tamaños medios de las EAPs (gráfico 2).

\section{Gráfico 1. Tamaño medio de las EAPs, valores absolutos (1988-2002-2008). Región Pampeana}

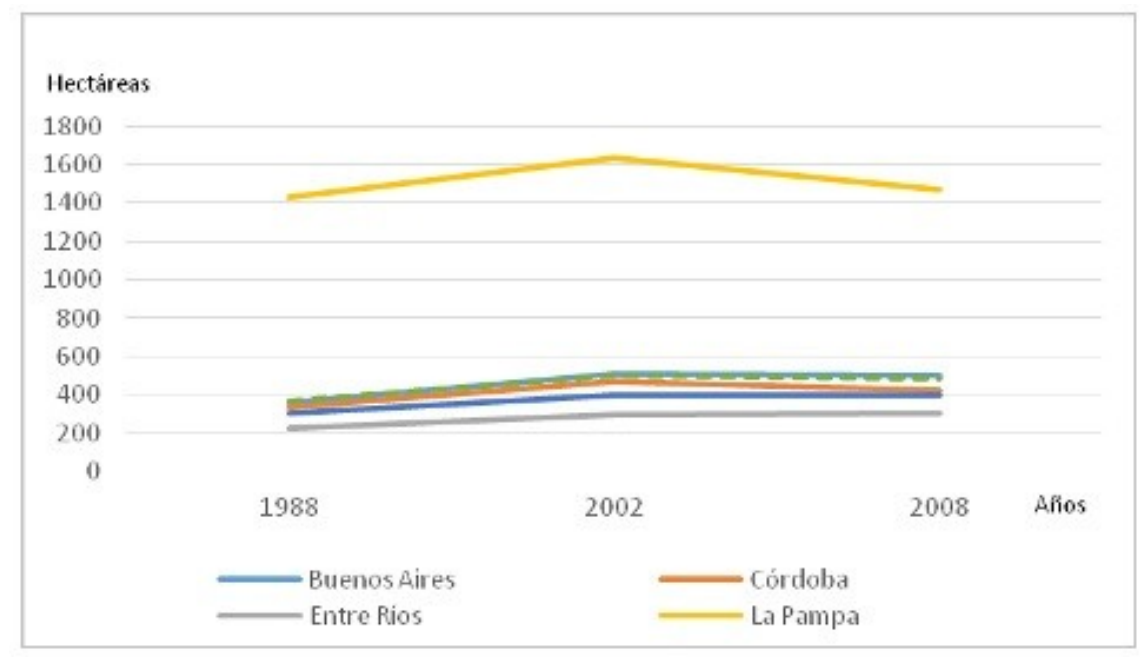

Fuente: Elaboración propia, CNA 1988, 2002 y 2008.

\section{Gráfico 2. Tamaño medio de las EAPs, valores absolutos (1988-2002-2008). NOA}

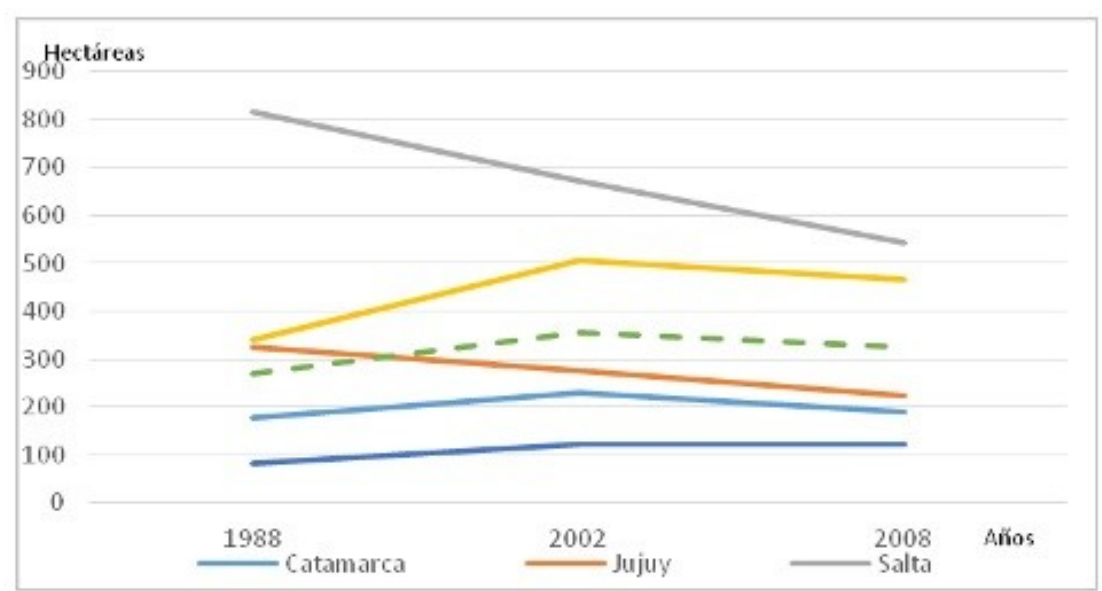

Fuente: Elaboración propia, CNA 1988, 2002 y 2008.

Las diferencias entre ambas regiones vienen dadas nuevamente por la magnitud de los cambios y la heterogeneidad de situaciones en el interior de las mismas. En el primer caso (la Región Pampeana) encontramos que el comportamiento en los 20 años analizados es bastante homogéneo; la principal diferencia no es la evolución de la variable sino la dimensión que presenta. Particularmente nos referimos al caso de la provincia de La Pampa, en donde el tamaño medio de las EAPs triplica al del resto de las provincias. En el caso del NOA, sin embargo, encontramos una gran heterogeneidad, con tendencias contrapuestas entre las provincias que componen la región.

\subsection{Composición de las exportaciones provinciales (2005-2011)}

Finalmente, el último elemento de este análisis es el de la composición de las exportaciones a nivel regional y 
provincial. Con respecto a la dinámica de la Región Pampeana y de las provincias que la integran, observamos un crecimiento constante hasta 2008 en las exportaciones de productos primarios (PP), manufacturas de origen agropecuario (MOA), manufacturas de origen industrial (MOI), y combustible y energía (COMB) (gráfico 3). La crisis internacional de 2009 significó una importante retracción en la exportación de todas las categorías analizadas, pero en los dos años siguientes ya los efectos de la crisis no son visibles, por el contrario, continúa un crecimiento estable en las exportaciones.

En el caso de los productos primarios exportados, observamos que sufren cierta retracción entre 2008 y 2009 para luego recuperar sus valores (18 \% del total de las exportaciones en 2005, $22 \%$ en 2011). Esto se debe a que, en un contexto de elevado crecimiento económico, la presión inflacionaria ejercida por el aumento de los precios internacionales de commodities desde mediados del año 2007 se exacerbó con el desabastecimiento interno inducido por el lockout de las asociaciones patronales del sector agropecuario durante el segundo trimestre del 2008, lo que generó un pico de inflación y un grado de incertidumbre política y macroeconómica inédita desde el año 2003 (Abeles, 2009).

Por su parte, las manufacturas de origen agropecuario se mantienen también constantes a lo largo del período, ya que varían del 37 al 38\% entre 2005 y 2011. Las manufacturas de origen industrial evidencian leves incrementos proporcionales, pasan de 34\% en 2005 a 38\% en 2011. En el conjunto de las MOI se destacan la automotriz, siderurgia e industria química, en ciertos casos desarrolladas por empresas multinacionales con presencia de filiales en el país. En cuanto a combustibles y energía, en el período informado se reduce de 9,4 \% a 3,2%.

\section{Grafico 3. Evolución de las exportaciones de Productos Primarios (PP), Manufacturas de Origen Agropecuario (MOA), Manufacturas de Origen Industrial (MOI), y Combustibles y Energía (COMB). Millones de dólares. (2005- 2011). Región Pampeana}

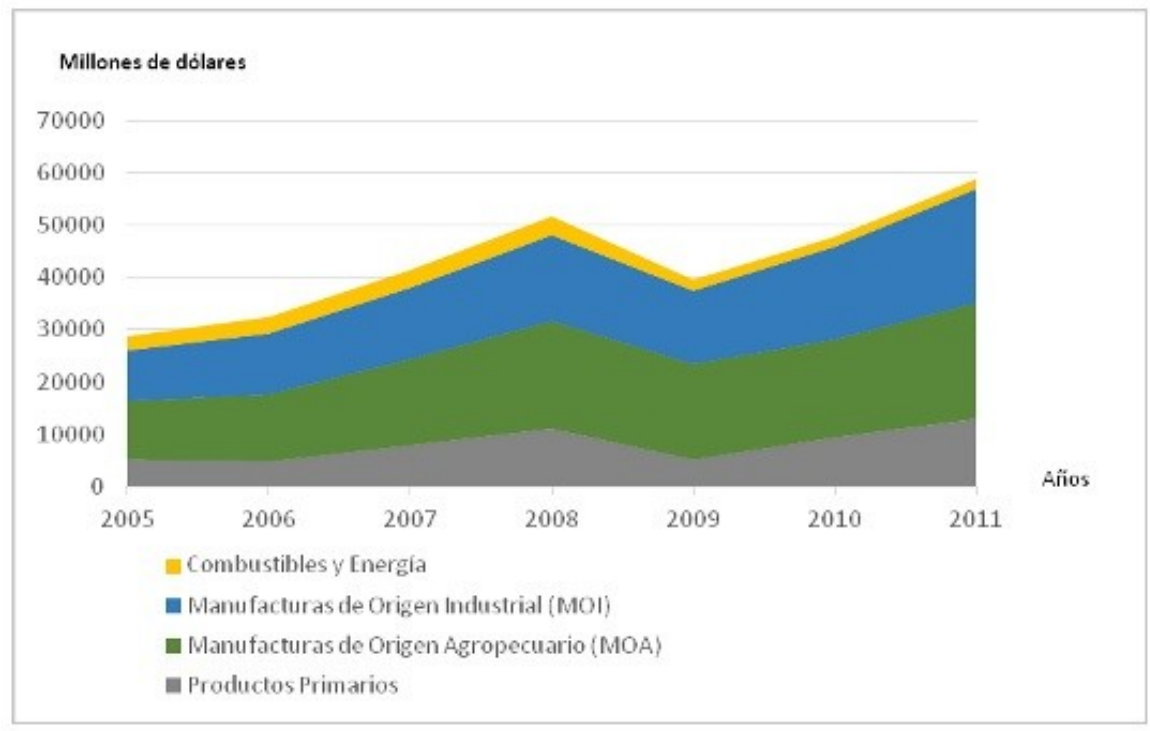

Fuente: elaboración propia sobre la base del SIIA.

Si tomamos ahora el caso del NOA, observamos que la composición de las exportaciones denota una estructura económica diferente. Como mencionamos antes, en la región fueron las actividades primarias las que históricamente motorizaron la economía y actualmente las áreas más dinámicas están representadas (además de por cereales y oleaginosas) por la minería (fundamentalmente de cobre, plata y oro), la citricultura, la caña de azúcar, la ganadería, la vitivinicultura, olivicultura y otras agroindustrias. El crecimiento de estas actividades explicaría el incremento de las exportaciones regionales operadas entre 2005 y 2011, el cual representa una variación positiva del 100 \% en el período analizado (gráfico 4). 
Gráfico 4: Reprimarización de la economía del NOA. Evolución de las exportaciones de Combustibles y Energía (Comb), Productos Primarios (PP), Manufacturas de Origen Agropecuario (MOA), y Manufacturas de Origen Industrial (MOI). Millones de dólares. Período 2005- 2011

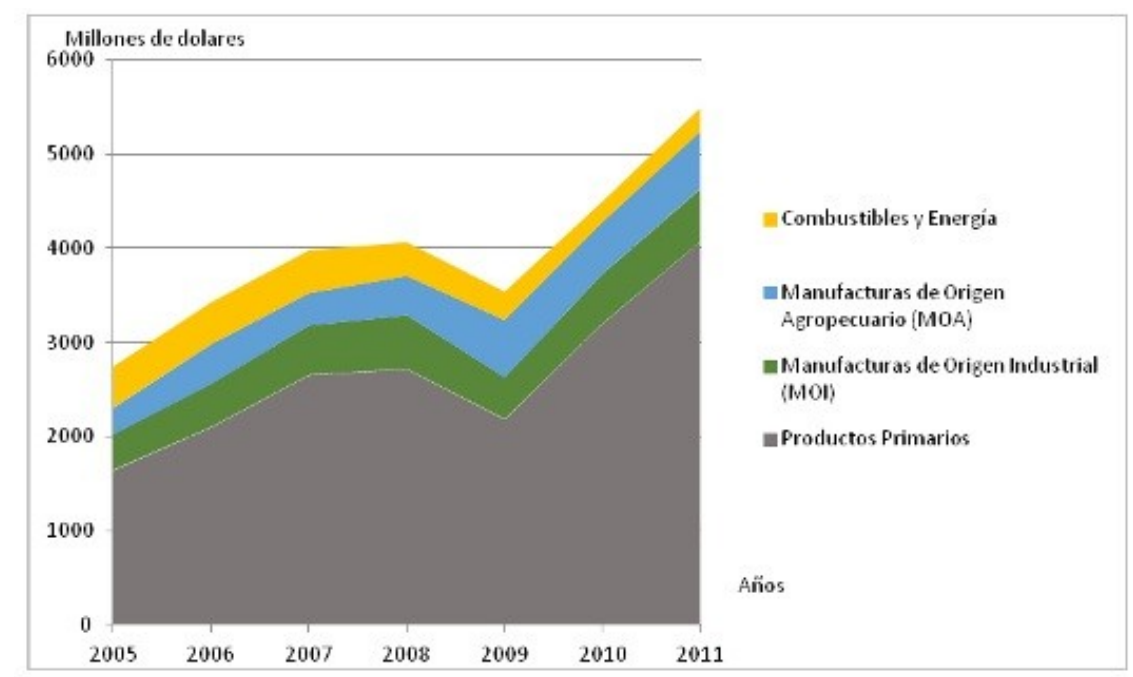

Fuente: elaboración propia sobre la base del SIIA.

Observamos entonces que, en 2005, casi el 60 \% de las exportaciones correspondían a productos primarios y en 2011 ese porcentaje se eleva hasta alcanzar el 74 \%, y es precisamente el incremento de las exportaciones primarias lo que explica casi la totalidad del incremento de las exportaciones. En este sentido, observamos que las Manufacturas de Origen Agropecuario (MOA), en 2005 significaron el 10,3 \% y terminaron en 2011 con el 11,1 \%; respecto a las Manufacturas de Origen Industrial (MOI), disminuyeron de 13,9 \% a 10,2 \%. Sin embargo, los que muestran una mayor disminución son los combustibles, los cuales pasan de representar el 16 \% al 4,6 \%.

Ahora bien, al discriminar el análisis a nivel provincial, cada una de ellas presenta comportamientos diferentes (Mapa 1).

Mapa 1: Exportaciones por provincias del Noroeste Argentino y Región Pampeana, 2011

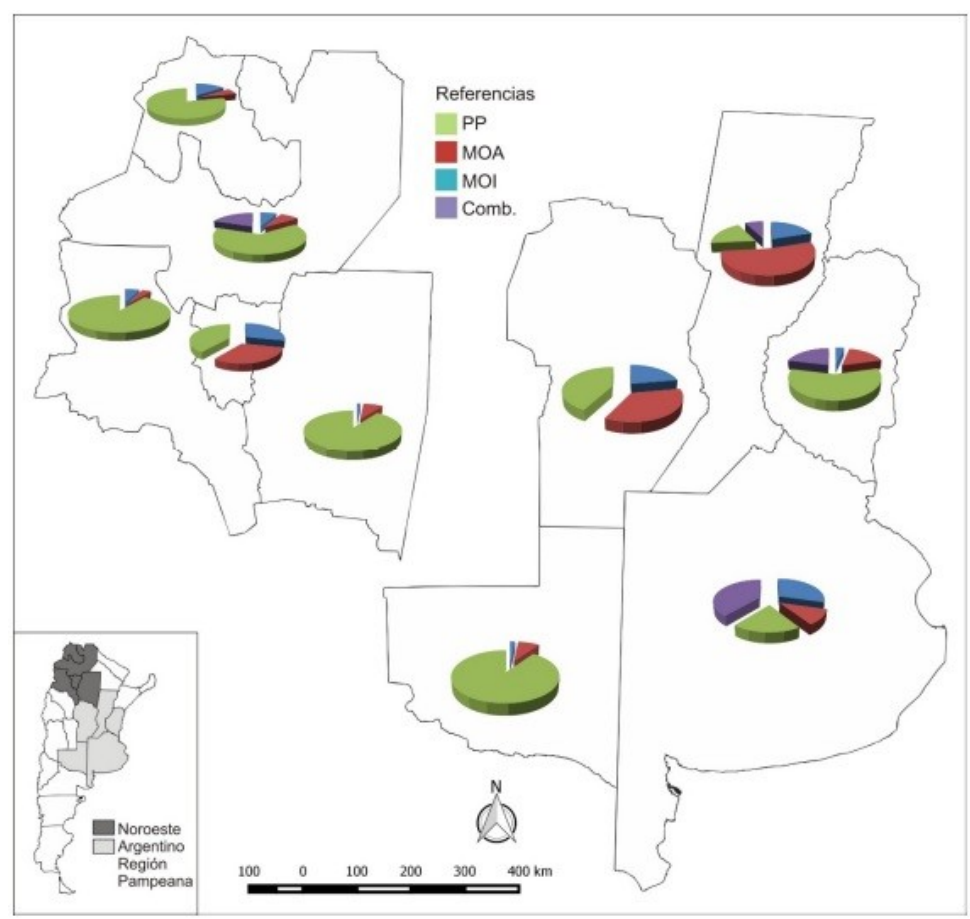


Con respecto a las exportaciones de productos primarios (PP) de la Región Pampeana, observamos que Buenos Aires implica el 46 \%, Córdoba el 25 \%, Entre Ríos el 8,9 \%, La Pampa el 2,5 \%, y Santa Fe el 18 \% en 2011, con incrementos en la primera unidad administrativa, reducciones en la segunda y estabilidad en las otras tres entre 2005 y 2011. Sobre las MOA, para el año 2011 Santa Fe denota la mayor proporción con un 50,5 \%, seguida de Buenos Aires con el 26 \%, luego Córdoba (20,7 \%), y con porcentajes mucho menores, Entre Ríos (2,6 \%) y La Pampa (0,2 \%). El comportamiento de las cinco provincias ha sido estable en todo el período de estudio.

Por otro lado, las MOI están representadas en un 67,5 \% por Buenos Aires, luego 18,9 \% Santa Fe, 13 \% Córdoba, 0,5 \% Entre Ríos y 0,1 \% La Pampa. Esto denota un cambio interesante en el período en cuanto a la variable espacial, dado que, de estar concentrado en un $80 \%$ en Buenos Aires hacia 2005, seis años más tarde evidenciamos mayor participación de Santa Fe y Córdoba. Finalmente, sobre combustibles y energía, el 89,4 \% es representado por Buenos Aires, el 7,4 \% por Santa Fe, y 3,2 \% por Entre Ríos. Como vemos, la Región Pampeana expresa estabilidad respecto de las exportaciones aunque con variaciones positivas sobre todo en las manufacturas de origen industrial.

Si hacemos una síntesis de lo observado en la Región Pampeana, podemos decir que es preponderante el rol de los productos primarios en La Pampa y Entre Ríos, y el de los productos manufacturados de origen agropecuario, en Santa Fe y Córdoba. Además, nos encontramos con una provincia como Buenos Aires que aparece definida como la más diversificada en cuanto a la participación de las cuatro variables de exportación puestas en análisis para 2011.

Con respecto al Noroeste Argentino, podemos observar el comportamiento diferencial en el interior de la región. Así, si consideramos los ingresos por exportaciones de MOI, vemos que es Tucumán la provincia que en 2011 aportaba más de la mitad de los ingresos regionales en este ítem (casi 300 millones de dólares), muy por arriba de las demás provincias, lo que implica también un porcentaje elevado de los ingresos por exportaciones a nivel provincial (28,6 \%). Mientras tanto, en las demás provincias, los ingresos por exportaciones de MOI no superaban el 7 \% de los ingresos provinciales en 2011. La situación se repite al considerar las MOA, respecto de las cuales todas las provincias excepto Tucumán (con un 31,7 \%) presentan porcentajes menores al $10 \%$.

Por el contrario, los ingresos por exportaciones de PP revelan gran importancia en la mayoría de las provincias, algunas de las cuales casi no exportan productos de otros ítems, como Catamarca (90,7 \%) y Santiago del Estero (90,7 \%). Por otro lado, Salta es la única provincia que exporta combustibles (con el 18,9 \%, valor nada insignificante dentro de sus exportaciones), lo que hace caer la participación de los PP dentro de sus exportaciones totales. Es decir, si no consideráramos las exportaciones de combustibles, Salta también podría catalogarse como una provincia exclusivamente exportadora de PP.

De acuerdo con el análisis de los datos presentados, podemos afirmar que las economías más primarizadas son las de Catamarca, Salta y Santiago del Estero, mientras la más diversificada es la de Tucumán.

En resumen, debemos destacar dos elementos entre las principales diferencias en relación con las regiones analizadas: por un lado, la magnitud de los valores considerados, y por otro la diversificación de la composición de las exportaciones en cada caso. Si consideramos el primer punto, para el NOA hablamos de valores 10 veces menores a los de la Región Pampeana, lo que da cuenta de la marginalidad de la economía del NOA en el contexto nacional. Mientras en la Región Pampeana los ingresos por exportaciones alcanzan casi los 60 mil millones de dólares, en el NOA no alcanzan los 6 mil millones. Y, en segundo lugar, como podemos ver en el mapa 1 , la composición de esas exportaciones en el caso de la Región Pampeana es altamente diversificada, mientras en el NOA asistimos en líneas generales a un proceso de reprimarización de la economía.

\section{Comentarios finales}

En este trabajo observamos que las diferencias entre las regiones analizadas son el resultado de legados históricos y geográficos, los cuales adquieren particular relevancia a partir de la consolidación de la globalización 
económica, política y cultural, y la aceleración del desarrollo científico y tecnológico. En este sentido, el contexto instaurado hacia 1990 -marcado por la incorporación de los llamados paquetes tecnológicos, exodirigidos, arribados de la mano de la apertura económica- dificultó el funcionamiento de la agricultura familiar, de los chacareros y de los campesinos en toda la república, y, en este caso particular, es posible afirmar, según los datos analizados en este trabajo sobre la evolución de la estructura agraria en la población rural del NOA y la Región Pampeana, la hipótesis de persistencia de las desigualdades.

Sostenemos que el fomento al extractivismo aumenta la desigualdad en la estructura agraria y vulnera a las poblaciones locales afectando principalmente a los indígenas, campesinos y agricultores familiares, lo cual manifiesta aquella "vulnerabilidad selectiva" a la que hacía referencia Harvey (2003). En un trabajo previo describimos cómo los efectos sociales que implican la pérdida de la tierra y los medios de vida para estas poblaciones pueden quedar soslayados ante el mejoramiento relativo de la calidad de vida que significó la distribución más equitativa de los ingresos generada por el estado en los últimos años (Krapovickas et al, 2017). Las políticas sociales en materia de vivienda, salud y educación, y la asignación universal por hijo han sido durante años la contracara del modelo extractivista y han servido para su justificación.

Es por esto que afirmamos que, a pesar de los esfuerzos de estas políticas en contener la pobreza, las desigualdades en torno al manejo de los recursos productivos en el agro argentino han empeorado. De esta forma, cuando se analizan las condiciones estructurales que en el mundo rural fueron causantes históricas de desigualdad -como el acceso a la tierra y las estructuras productivas- se constata la persistencia de las tendencias concentradoras de la riqueza.

Observamos entonces la progresiva reducción en la cantidad de unidades productivas y el incremento en sus tamaños medios, en paralelo con un proceso de reprimarización de la economía, especialmente en la región del NOA, fenómeno que, como señalan Barsky y Gelman (2005, p. 386), es resultado de la exposición de los productores a las contingencias del mercado internacional de cereales y oleaginosas que se caracteriza por su extrema movilidad.

De este modo, en las dos regiones analizadas y para el período 1988-2008, podemos observar que el número de EAPs disminuye desde algo más de 250 mil a 160 mil unidades, lo que significa una variación de $-34 \%$. A su vez, si analizamos la incidencia de las EAPs con límites definidos y sin límites definidos en cada una de las regiones, encontramos que las primeras se redujeron mayormente en la Región Pampeana (-40\%), mientras que las segundas mostraron una mayor caída en el NOA (-46 \%).

Sin embargo, a pesar de la diversidad de situaciones existentes, entre las regiones y en interior de cada una de ellas, encontramos tendencias que las aproximan en cuanto a la evolución de las principales variables relativas a la estructura agraria. No sucede lo mismo, en cambio, respecto de la composición de los ingresos por exportaciones, ya que mientras la Región Pampeana mantiene a lo largo de la década una estructura económica diversificada y sostenida en la variedad de productos y manufacturas exportadas, en el NOA observamos un cambio en la estructura económica, con un claro proceso de reprimarización. Entonces, podemos afirmar que se evidencia la persistencia de las desigualdades regionales, situación que no sólo no se ha modificado sustancialmente, sino que parece haberse profundizado.

\section{Notas}

1 El criterio de regionalización que rige este trabajo se basa en los principios de organización que han dado los propios estados provinciales. La Reforma Constitucional de 1994, a través del artículo 124, faculta a las provincias a "crear regiones para el desarrollo económico y social y establecer órganos con facultades para el cumplimiento de sus fines” y a partir de esa fecha se han suscripto una serie de tratados interprovinciales que tienden a institucionalizar la existencia de ámbitos geográficos con especificidades que se han plasmado a partir de largos procesos históricos. Las regiones resultantes son cinco: NOA (Noroeste Argentino), NEA (Noreste 
Argentino), Nuevo Cuyo, Patagonia, y Centro y Buenos Aires (Ministerio de Economía y Finanzas Publicas, 2011). También se han contemplado las regionalizaciones establecidas por el INDEC y trabajadas por autores como Velázquez (2016). En referencia a estos últimos es que incorporamos a La Pampa dentro de nuestra llamada Región Pampeana.

$\underline{2}$ La recolección de datos afectó fundamentalmente a la Región Pampeana y principalmente a la provincia de Buenos Aires (se calcula que dejaron sin censar en esa provincia 10 millones de hectáreas, la mitad aproximadamente de la estimación de las hectáreas no censadas en todo el país) y hasta el propio INDEC reconoció el fracaso del Censo (INDEC, 2009; Giarracca, 2009). Sin embargo, en la región del NOA y en provincias como Santa Fe, se cubrieron altos porcentajes de las superficies censadas en 2002 (ver tabla 2 ). Al tratarse este trabajo sobre una comparación regional además de una evolución histórica, decidimos igualmente incorporar los datos del Censo 2008 y evaluamos los resultados críticamente.

$\underline{3}$ Siguiendo a Giarracca y a Teubal (2013) entendemos como reprimarización de la economía al énfasis puesto sobre la producción de materias primas que se constituyen en productos básicos de exportación (commodities), y hablamos de reprimarización porque en algún momento de nuestra historia tuvimos vocación industrializadora.

$\underline{4}$ El año 1998 se constituyó en una bisagra en cuanto al crecimiento de la superficie cosechada con soja y la caída de la superficie de algodón. Esto se dio a causa de diferentes factores, en primer lugar, la suba de precios internacionales de la soja que permitió la rentabilidad de las inversiones que implicaba el desmonte, en segundo lugar, la necesidad de expansión de capitales principalmente pampeanos y, en tercer lugar, la habilitación del poder político local, con sus irregularidades en la entrega de tierras (Bageneta, 2015).

$\underline{5}$ En la provincia de Santiago del Estero se reconocía una práctica sistemática de entrega de tierras a empresarios provenientes, en su mayoría, de la región pampeana, quienes presionaban a los pobladores locales, muchos de los cuales presentan tenencias de tierra precarias (De Dios, 2006, 2010; Paz, 2010; citado por Bageneta, 2015:116).

\section{Bibliografía}

Abeles, M. (2009). El impacto de la crisis internacional en la economía argentina. Revista de Trabajo, 7, $185-212$. Recuperado de http://www.trabajo.gob.ar/left/estadisticas/descargas/revistaDeTrabajo/2010n07 revistaDeTrabajo/2010n07 a09 mAbeles.pdf

Amarante, V., y Jiménez, J. P. (2015). Desigualdad, concentración y rentas altas en América Latina. En J.P. Jiménez (ed.), Desigualdad, concentración del ingreso y tributación sobre las altas rentas en América Latina (pp. 13-47). Libros de la CEPAL Nº 134 (LC/G.2638-P), Santiago de Chile: CEPAL.

Bageneta, J. M. (2015). Del algodón a la soja. Territorio, actores y cooperativas en el Gran Chaco Argentino (1960-2010). Buenos Aires: Editorial Intercoop. Recuperado de http://www.biblioteca.coop/bdc/libros/Delalgodon-a-la-soja/index.html

Balsa, J. (2006). El desvanecimiento del mundo chacarero: transformaciones sociales en la agricultura bonaerense 1937-1988. Buenos Aires: Universidad Nacional de Quilmes.

Bárcena Ibarra, A., y Prado, A. (2016). El imperativo de la igualdad: por un desarrollo sostenible en América Latina y el Caribe. Buenos Aires: CEPAL, Siglo XXI Editores.

Barsky, O., y Gelman, J. (2005). Los cambios en las políticas macroeconómicas y su impacto sobre el sector agropecuario 1970-2000. En O. Barsky y J. Gelman, Historia del agro argentino. Desde la conquista hasta fines del siglo XX (pp. 360-406). Buenos Aires: Mondadori. 
Bebbington, A. \& Humphreys Bebbington, D. (2011). An Andean Avatar: Post-Neoliberal and Neoliberal Strategies for Securing the Unobtainable. New Political Economy, 16(1), $131-145 . \quad$ Doi: http://dx.doi.org/10.1080/13563461003789803

Blanco, M. (2005). Argentina: la incorporación de la agricultura conservacionista en la región pampeana. Debate agrario, 38, 141-157. Recuperado de www.cepes.org.pe/debate/debate38/06Blanco.pdf

Bolsi, A., y Paolasso, P. (comp) (2009). Geografía de la pobreza en el Norte Grande Argentino. Tucumán: Programa de las Naciones Unidas para el Desarrollo, UNT.

Bolsi, A. (1997). El Noroeste Argentino: una aproximación a su mundo agrario. En A. Bolsi (comp.), Problemas Agrarios del Noroeste Argentino (contribuciones para su inventario) (pp. 181-183). Tucumán: Universidad Nacional de Tucumán - Junta de Andalucía.

Borras, S. M. Jr., Kay, C., Gómez, S., \& Wilkinson, J. (2012). Landgrabbing and global capitalist accumulation: keyfeatures in Latin America. Canadian Journal of Development Studies, 33(4), $402-416 . \quad$ Doi: http://dx.doi.org/10.1080/02255189.2012.745394

Comisión Económica para América Latina y el Caribe (2016). Horizontes 2030: la igualdad en el centro del desarrollo sostenible. Santiago de Chile: CEPAL.

Domínguez, D., y Sabatino, P. (2006). Con la soja al cuello: crónica de un país hambriento productor de divisas. En H. Alimonda (comp.). Los tormentos de la materia. Aportes para una ecología política latinoamericana (pp. 213-238). Buenos Aires: Consejo Latinoamericano de Ciencias Sociales.

Gaignard, R. (1989). La Pampa Argentina. Ocupación-poblamiento-explotación, de la conquista a la crisis mundial (1550-1930). Buenos Aires: Solar, Dimensión Argentina.

García, M., Gonzalez, M. del C., y Román, M. (2016). Una visión exploratoria del desmonte para distintos tipos de productores en tres departamentos de la provincia de Santiago del Estero. En M. Román y M. González, del C. (coord.) Transformaciones agrarias argentinas durante las últimas décadas. Una visión desde Santiago del Estero y Buenos Aires (pp. 103-124). Ciudad Autónoma de Buenos Aires: Editorial Facultad de Agronomía.

Giarracca, N. (comp.) (2001). ¿Una Nueva Ruralidad en América Latina? Buenos Aires: Consejo Latinoamericano de Ciencias Sociales.

Giarracca, N. (2009). El fracaso del censo agropecuario. Crítica de la Argentina. Buenos Aires. Recuperado de http://www.ger-gemsal.org.ar/el-fracaso-del-censo-agropecuario/

Giarracca, N., y Teubal, M. (2013). Introducción. En M. Teubal y N. Giarracca, Actividades extractivas en expansión: ¿Reprimarización de la economía argentina? (pp. 9-18). Buenos Aires: Antropofagia.

Giarracca, N., y Teubal, M. (2013). Las actividades extractivas en Argentina. En M. Teubal, y Giarracca, N., Actividades extractivas en expansión: ¿Reprimarización de la economía argentina? (pp. 19-44). Buenos Aires: Antropofagia.

Giberti, H. (2003). Modernizado e insatisfactorio sector agropecuario. Realidad Económica, 200, 103-128.

Göbel, B., Góngora-Mera, M., y Ulloa, A. (2014). Las interdependencias entre la valorización global de la naturaleza y las desigualdades sociales: abordajes multidisciplinarios. En B. Göbel, M. Góngora-Mera y A. Ulloa (ed.), Desigualdades socioambientales en América Latina (pp. 13-46). Bogotá: Universidad Nacional de Colombia. Berlín: Ibero-Amerikanisches Institut.

Gras, C. (2012). Cambio agrario y nueva ruralidad: Caleidoscopio de la expansión sojera en la región pampeana. Trabajo y sociedad, 18(XV), 7-24. Recuperado de http://www.scielo.org.ar/scielo.php? script=sci arttext\&pid=S1514-68712012000100001 
Gras, C. (2006). Actividades, ingresos y relaciones sociales implicadas en la pluriactividad”. En G. Neiman y C. Craviotti (comp.), Entre el campo y la ciudad. Desafíos y estrategias de la pluriactividad en el agro (pp. 161183). Buenos Aires: CICCUS.

Harvey, D. (2003). Espacios de Esperanza. Madrid: Akal.

Harvey, D. (2004). Nuevo imperialismo: acumulación por desposesión. En L. Panitch y C. Leys (ed.), El nuevo desafío Imperial (pp. 95-126). Buenos Aires: Consejo Latinoamericano de Ciencias Sociales.

INDEC (2009). Censo Nacional Agropecuario 2008, CNA'08. Situación del operativo, Resultados anticipados. Buenos Aires: Instituto Nacional de Estadística y Censos. Recuperado de: http://www.indec.gov.ar/nuevaweb/cuadros/11/nota cna08 08 09.pdf.

Kay, C. (2007). Algunas reflexiones sobre los estudios rurales en América latina. Revista de Ciencias Sociales, 29, Facultad Latinoamericana de Ciencias Sociales-Sede Académica de Ecuador. Recuperado de www.flacso.org.ec/docs/i29kay.pdf

Krapovickas, J. (2009). Cambio socio ambiental en el Chaco argentino y su relación con la expansión de la soja en la década del 90 (Tesis de maestría). Universidad Autónoma de Barcelona, Barcelona, España.

Krapovickas, J. (2016). El extractivismo sojero y sus consecuencias humanas. Modelos de desarrollo en disputa en el Chaco Argentino. Revista Alternativa, 5, 114-139. Recuperado de http://ppct.caicyt.gov.ar/index.php/alternativa/article/download/9446/pdf

Krapovickas, J., Mikkelsen, C. y Garay, A. (2017). Lo rural fragmentado. Evidencias en el Noroeste Argentino y la Región Pampeana. En P. Paolasso, F. Longhi y G. Velázquez, (comp.). Desigualdades y Fragmentación Territorial en la Argentina durante la primera década del siglo XXI. Buenos Aires: Imago Mundi.

Krapovickas, J., Sacchi, L. y Hafner, R. (2016). Firewood supply and consumption in the context of agrarian change: the North Argentine Chaco from 1990 to 2010. International Journal of the commons, 10(1), $220-243$. doi: http://doi.org/10.18352/ijc.609.

Lattuada, M. y Neiman, G. (2005). El campo argentino: crecimiento con exclusión. Buenos Aires: Capital Intelectual.

Manzanal, M. (1995). Globalización y ajuste en la realidad regional argentina: ¿Reestructuración o difusión de la pobreza? Realidad Económica, 134, 67-82.

Massuh, G. (ed.). (2012). Renunciar al bien común. Extractivismo y (pos)desarrollo en América Latina. Buenos Aires: Mardulce.

Ministerio de Economía y Finanzas Públicas (2011) Regionalización: nota metodológica. Consultado el 27 de abril de 2017. Recuperado de: http://www.mecon.gov.ar/peconomica/dnper/reg nota meto.pdf

Morina, O. y Cacace, G. (2010). Agro capitalismo extractivo y depredadores en una geografía periférica: la Argentina del Bicentenario. En O. Morina (ed., dir. y comp.), Geografías de la Agricultura Industrial sojera en la Argentina ¿viaje de ida? (pp. 19-46). Luján: Departamento de Ciencias Sociales. Programa de Estudios Geográficos (PROEG).

Neiman, G. (2010). Los estudios sobre el trabajo agrario en la última década. Una revisión para el caso argentino. Mundo agrario, 10(20), 1-20. Recuperado de http://www.mundoagrario.unlp.edu.ar/rt/printerFriendly/v10n20a20/480

Osatinsky, A. (2009). Pobreza y transformaciones económicas en Catamarca y Tucumán (1980-2002). Población y sociedad, 16(2), 187-222. Recuperado de http://www.scielo.org.ar/scielo.php?script=sci arttext\&pid=S1852$\underline{85622009000200001}$

Paz, R. y Jara, C. (2014). Estructura agraria en Santiago del Estero: el proceso de territorialización de las 
explotaciones campesinas sin límites y su tensión frente al avance del capitalismo agrario. Estudios rurales,1(6), 81-99. Recuperado de http://ppct.caicyt.gov.ar/index.php/estudios-rurales/article/view/1910

Programa de las Naciones Unidas para el Desarrollo, PNUD (2010). Informe regional sobre desarrollo humano para América Latina y el Caribe 2010: actuar sobre el futuro: romper la transmisión intergeneracional de la desigualdad. San José (Costa Rica): PNUD. Recuperado de hdr.undp.org/sites/default/files/rhdr-2010-rblac.pdf

Reboratti, C. (2006). La Argentina rural entre la modernización y la exclusión. En A. Geraiges de Lemos, M. Arroyo y M. L. Silveira, América Latina: ciudades, campo e turismo (pp. 175-187). San Pablo: Consejo Latinoamericano de Ciencias Sociales.

Reboratti, C. (2007). El espacio rural en América Latina: procesos, actores, territorios. En M. V. Fernández Caso y R. Gurevich (coord.). Geografía. Nuevos Temas, nuevas preguntas. Un temario para la enseñanza (pp. 97-124). Buenos Aires: Biblos.

Reboratti, C. Prudkin, N. y León, C. (1996). ¿Pampeanización del NOA o la adaptación al ecosistema local? En M. Manzanal (comp.), El desarrollo rural en el noroeste argentino -antología (pp. 161-168). Salta: Proyecto Desarrollo Agroforestal en comunidades rurales del Noroeste Argentino.

Rivas, A. I. y Natera Rivas, J. J. (2009). La distribución de la tierra en el Norte Grande Argentino: persistencias y cambios. Baetica, 31, 91-113. Recuperado de http://riuma.uma.es/xmlui/handle/10630/6523

Rivas, A. I. (2013). Irrupción del modelo sojero en el ambiente oriental de la provincia de Tucumán. En M. Teubal y N. Giarracca, Actividades extractivas en expansión ¿Reprimarización de la economía argentina? (pp. 119-136). Buenos Aires: Antropofagia.

Román M., González, M. del C. y Lipshitz, H. (2016). Intensificación agrícola o expansión de la frontera. Un análisis comparativo entre Buenos Aires y Santiago del Estero. En M. Román y González, M. del C. (coord.), Transformaciones agrarias argentinas durante las últimas décadas. Una visión desde Santiago del Estero y Buenos Aires (pp. 1-20). Buenos Aires: Editorial Facultad de Agronomía.

Shmite, S. M. (2008). Chacarero: ¿un viejo concepto para un nuevo sujeto agrario? En Décimo Encuentro Internacional Humboldt, llevado a cabo en Rosario, Santa Fe, 13 al 17 de octubre de 2008.

Smith, N. (2006). La geografía del desarrollo desigual. En B. Dunn y H. Radice, (eds.), 100 years of permanent revolution: Results and prospects (pp. 180-195). Londres: Pluto press.

Svampa, M. (2011). Pensar el desarrollo desde América Latina. En Seminario Latinoamericano "Derechos de la Naturaleza y Alternativas al extractivismo. Seminario co-organizado por Colectivo Voces de Alerta, CLAES, Jóvenes por la Igualdad y CEPPAS. Buenos Aires, noviembre de 2011.

Svampa, M. y Antonelli, M. A. (2009). Minería transnacional, narrativas del desarrollo y resistencias sociales. Buenos Aires: Editorial Biblos.

Velázquez, G. (director) (2016). Geografía y Calidad de Vida. Análisis Regional y Departamental (2010). Tandil:

UNCPBA. Recuperado de

https://www.researchgate.net/publication/301290773 Geografia y calidad de vida en Argentina 2010 